\title{
X10, In-Home Prehabilitation Increases Post-Surgical Range of Motion, Quadriceps and Calf Strength
}

\author{
Robert J Ference ${ }^{1}$ and Carl Freeman ${ }^{2 *}$ \\ ${ }^{1}$ Harper University Hospital, Detroit, USA \\ ${ }^{2}$ Department of Biological Sciences, Wayne State University, USA
}

*Corresponding author: D. Carl Freeman, Department of Biological Sciences, Wayne State University, 2955 Quail Run Dr. Troy, USA.

\author{
Received Date: May 08, 2020
}

Published Date: May 26, 2020

\begin{abstract}
The efficacy of prehabilitation, for knee surgery, is controversial. Meta analyses show that range of motion is improved, but not quadriceps or hamstring strength. Here, we evaluate prehabilitation using the newly patented, computerized, in-home, X10 knee recovery system for both prehabilitation and rehabilitation. We retrospectively examined 201 patients at multiple time points for range of motion (extension, flexion), quadriceps, hamstring, and calf strength between the intervention group $(\mathrm{n}=125)$ which used the $\mathrm{X} 10$ for both prehabilitation $(2-3$ weeks) and rehabilitation (3-4 weeks)and the control group $(\mathrm{n}=76)$ which used the X10 for only rehabilitation (3-4 weeks). Range of motion was measured by the X10 machine using an ultrasensitive inclinometer and strength was measured as pressure applied to a load cell. We also compared X10 prehabilitation and rehabilitation to standard physical therapy using a literature study (Calatayud et al. 2017).

Prior to surgery, prehabilitation significantly improved extension, $8.0^{\circ}\left(5.8^{\circ}\right)$ mean (standard deviation), flexion $12.1^{\circ}\left(12.3^{\circ}\right)$, range of motion $16.0^{\circ}\left(16.7^{\circ}\right)$; quadriceps strength $5.8(5.4) \mathrm{kg}$, hamstring strength $2.5(2.3) \mathrm{kg}$, and calf strength 11.2 (9.3) kg. Post-surgically, X10 prehabilitation increased flexion $120.7^{\circ}\left(10.7^{\circ}\right)$ vs $113.8^{\circ}\left(13.0^{\circ}\right)$, range of motion, $120.4^{\circ}\left(11.6^{\circ}\right)$ vs $112.9^{\circ}\left(11.7^{\circ}\right)$. X10 prehabilitation improved quadriceps strength 5.5\%, while the non-prehabilitation group lost $17.1 \%$. Calf strength improved in the prehabilitation group by $30.5 \%$, but only $0.2 \%$ in the control group. Hamstring strength was unchanged. Compared to standard physical therapy, X10 prehabilitation and rehabilitation were superior.
\end{abstract}

Keywords: Knee, Prehabilitation, X10, Total knee arthroplasty

Abbreviations: RoM: Range of motion; NIMS: Neuromuscular stimulations, KG: kilogram, ANOVA: Analysis of Variance; TUG: Timed up and go test; NS: Not Significant

\section{Introduction}

Joint replacement is a terminal treatment for end-stage knee and hip osteoarthritis. Osteoarthritis is usually a multiyear diseaseand decreases patients' quality of life by decreasing mobility, functionality, conditioning and increasing pain [1]. It is, reasonable to ask if exercising before surgery might, either shorten the time it takes patients to recover (reach benchmarks) or improve the patient outcomes. Fortin et al. [2] report that the preoperative condition of the leg is a reliable predictor of patients' outcome six months post-surgery. The issue then becomes: Does increasing the presurgical range of motion (RoM) or strength of patients demonstrably improve patients' post-surgical outcomes?
Despite the allure of prehabilitation, its efficacy remains contentious [3-6] Some of the discrepancies might be due to the nature of the interventions which range from neuromuscular stimulations (NIMS) [7] to home exercises [8] to the use of weights and machines [1,4]. Data interpretation is also obscured by the wide range of duration of prehabilitation tests ranging from three or four weeks $[7,9]$ to 12 weeks $[1,4,7,8,9]$. Even when interventions are similar, the results vary $[3,4]$. A recent meta-analysis found that, in general, prehabilitation shortened hospital stays, and increased performance for range of motion and the sit-to-stand test but not for quadriceps strength or the six-minute walk [10]. Part of the 
problem of intensive prehabilitation is that patients are being asked to exercise already painful joints [1]; in some cases, by applying asubstantial torque to the joint [4].

Here, we evaluate a new intelligent knee prehabilitation and rehabilitation machine, the X10 (Figure 1), [11] with specific exercises that do not apply a substantial torque to the knee. The X10 is used to increase both range of motion and strength (quadriceps, hamstring, calf). The X10 has patented pressure sensors that monitor whether or not the pressure being applied is below the maximum level set by the patient. Rather than exceed the maximum pressure, as patients exercise, the machine stops when it reaches the patient's preset level, and reverses; thus, protecting the patient from pain. Because the X10 causes minimal controllable levels of pain and is used in the home, patients can utilize the machine multiple times a day (usually 3 times) beginning the day after surgery. The onboard computer texts the results of each session to a HIPPA compliant server and the data are then made available to surgeons and telemedicine coaches, who oversee the patients' recovery and can respond in real time to patients' questions and needs. Surgeons can then discharge patients when they reach appropriate benchmarks rather than the maximum allowable authorized visits. Accordingly, the X10 represents a new generation of therapeutic device [Figure 1].

Figure 1: Patient on the $\mathrm{X} 10$.

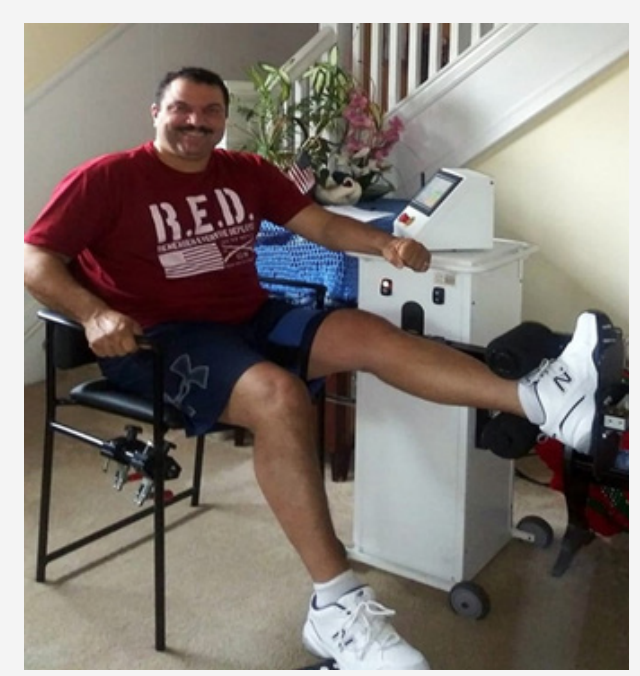

\section{Material and Methods}

We retrospectively (3/17-12/18) examined the data from 201 patients, 125 used the X10 for both prehabilitation (2-3 weeks) and rehabilitation (usually 3-4 weeks), and 76 used the device for only rehabilitation (3-4 weeks). The latter group served as the control. We also assessed patient age, gender, and BMI. All patients provided informed consent.

The X10 has a single actuator arm which is computer controlled and uses pressure either to move or resist movement the leg. Accordingly, the machine can be used to increase both the passive and active range of motion (RoM) and it can also be used to exercise the quadriceps, hamstrings, and calf muscles. The X10 is customized to each patient when it is delivered to their home. During the initial set-up the patients are instructed on how to use the machine and assigned a telemedicine coach who will oversee their recovery. Thereafter, the patients control the machine. Patients are instructed to use the machine three times a day for RoM and every other day for strengthening during prehabilitation.

The machine is capable of doing isometric, concentric, and eccentric exercises. Here, we report on extension, flexion, passive RoM, and isometric strength $(\mathrm{kg})$ of the calf, hamstring and quadriceps muscles. While the literature typically reports on both the hamstring and quadriceps strength, we also encourage patients to exercise their calves to reduce swelling rapidly. When patients are engaged in strength exercises the arc through which the machine works is about $15^{\circ}-30^{\circ}$ so that the leg is kept fairly straight and the joint is not subjected to great torque.

To assess patients' functional performance, we conducted the Timed Up and Go test (TUG) in which patients are timed as they rise from an arm chair, walk 10 feet, turn around, walk back to the chair and sit down. Because the X10 is a recent innovation, we have analyzed its accuracy (appendix) we have also compared the results of our study to those of Calatayud et al. 4, who report on intensive prehabilitation using standard physical therapy equipment. Like us, they examined outcomes at baseline, pre-surgery, and 30 days post-surgery for extension, flexion, quadriceps strength, hamstring strength and the TUG test. As Calatayud et al. [4] did not report the standard deviations for their measurements, we cannot do a statistical comparison. Instead they report the 95\% confidence interval; we have similarly reported the confidence interval when comparing our work to theirs, if the confidence intervals do not overlap, we can infer that the difference is statistically significant. 
Below, we briefly outline the strength exercises.

- Quadriceps Strengthening: knee extension into footplate with simultaneous hip flexion to tolerance, repeatedly for 30 seconds, 3 sets as tolerated (isometric) at a $60^{\circ}$ angle.

- Hamstring Strengthening: knee flexion into back roller pad to tolerance, repeatedly for 30 seconds, 3 sets, each as tolerated (isometric) $60^{\circ}$ angle

- $\quad$ Calf Strengthening: ankle plantar flexion into footplate to tolerance, relax into dorsi flexion, repeatedly for 30 seconds, 3 sets, each as tolerated (active motion) 15 degree angle.

Rowe [12] recommends using $110^{\circ}$ of flexion as an indicator of the ability to perform the activities of daily living, we have chosen to use a slightly more stringent measure, $110^{\circ}$ of RoM as our indicator of the patients' ability to engage in the activities of daily living.

\section{Statistical Analyses}

When comparing two means, we used a Student's t test. For before and after comparisons we used a paired t test. We used a repeated-measures analysis of variance (ANOVA) when we compared the two treatment groups using their baseline and final measurements. This was done for the strengthening exercises and the TUG test. Specifically, we looked for a time $\mathrm{x}$ treatment interaction to determine if the control and treatment group had the same response over time.

\section{Result}

\section{Demography}

The mean age for patients in the prehabilitation group (59.7 (5.3) years)differed significantly from the control group (63.5 (8.6) years $)(t=3.98, P<0.001)$. Younger patients tend to perform worse
[13] so actually the prehabilitation patients are at a disadvantage. There was no difference in the mean BMI between the intervention group (31.7 (6.3)) and the control group (30.9 (6.1)), $(t=0.92$, N.S.). Similarly, there was no difference in the gender between the prehabilitation (57 males, 68 females) and the control group (37 males, 39 females) (X2 $=0.18$, N.S.).

\section{Before surgery}

\section{Extension}

Fifty-two of the patients had perfect $\left(0^{\circ}\right)$ extension at their baseline, and accordingly could make no progress. As we wanted to see if the X10 made a difference in prehabilitation, we have excluded these from the analysis. In table 1 we report on the mean difference in extension of the patients that had nonzero extension on the first day. The average patient improved their extension by $8.0^{\circ}\left(5.8^{\circ}\right)(\mathrm{P}<0.001)$.

\section{Flexion}

The X10 has a maximum flexion of $130^{\circ}$. Thirty-six patients began with perfect flexion at their baseline evaluation, and thus could make no improvement. We have examined the flexion of the 89 patients who had less than $130^{\circ}$ flexion (table 1 ). Clearly, those patients who could, improve their flexion, did so.

\section{Range of Motion}

Twenty-six patients began with the maximum measurable RoM $\left(130^{\circ}\right)$; we excluded these from the analysis and computed the average RoM for the remaining 99 patients, as these were the only patients who could improve their RoM. Patients who were not at the maximum RoM, to start with, experienced, on average, asignificant increase during prehabilitation(mean $16.0^{\circ}\left(16.7^{\circ}\right)$ ) (Table 1).

Table 1: Here, we compare the extension, flexion range of motion and strength of patients before they began exercises (baseline) to their final strength measurement before surgery to judge where or not prehabilitation was efficacious in increasing strength. Strength improved significantly for all three muscle groups. For the RoM measurements we used only those patients who did not begin with a perfect score to see if the X10 made a difference.

\begin{tabular}{|c|c|c|c|c|c|c|}
\hline Parameter & Unit & Mean Difference & Std. Deviation & $\mathrm{t}$ & df & $P$ \\
\hline Extension & ○ & 8.0 & 5.8 & 11.70 & 72 & 0.000 \\
\hline Flexion & $\circ$ & 12.1 & 12.3 & 9.59 & 95 & 0.000 \\
\hline Range of Motion & $\circ$ & 16.0 & 16.7 & 10.32 & 116 & 0.000 \\
\hline Quadriceps & $\mathrm{kg}$ & 5.8 & 5.4 & 11.77 & 120 & 0.000 \\
\hline Hamstring & $\mathrm{kg}$ & 2.5 & 2.3 & 11.81 & 118 & 0.000 \\
\hline Calf & $\mathrm{kg}$ & 11.2 & 9.3 & 12.86 & 114 & 0.000 \\
\hline
\end{tabular}

\section{Strength}

Patients were asked to use the X10's strengthening modules once a day, every other day during prehabilitation to strengthen their quadriceps, hamstring and calf muscles. While measurements were taken every time the patient used the machine, here we report the baseline and last measurement (Table 1) so that our results are comparable to those reported in the literature $[1,4]$. There was a significant increase in strength for each of the three muscle groups (table 1).

\section{After Surgery with and without prehabilitation}

\section{Extension}

There was no functional difference between the two groups at 30 days post-surgery (mean for prehabilitation, 0.2 (1.7); mean for non-prehabilitation $0.7(2.5))$. 


\section{Flexion}

The difference between prehabilitation and nonprehabilitation patients increased with time. Patients who engaged in prehabilitation had significantly greater flexion at 30 days postsurgery (mean 6.9 $)(\mathrm{t}=4.10, \mathrm{P}<0.005)$.

\section{Range of Motion}

Prehabilitation patients start out with a slight advantage which grows over time. At day 30, the average difference is highly significant $8.5^{\circ}$ (F 1,199 $=13.73, \mathrm{P}<0.001$ ) (Figure 2). But this difference does not tell the whole story. In figure 3we plot the cumulative percentage of patients with a RoM greater than or equal to $110^{\circ}$ One hundred ten degrees was used as an indicator of the ability to perform the activities of daily living [11]. The relatively modest difference in the average RoM, translates into a substantial difference in the cumulative percentage of patients with a RoM greater than $110^{\circ}$. There are two important points that should be mentioned about figure 3; the first is the $33 \%$ difference in the percentage of patients who reach $110^{\circ}$ or better RoM. The second important feature is that curves flatten indicating that the percentage of patients who reached this benchmark is not increasing after 30 days, i.e., the patients who have not reached the benchmark in 30 days will likely not reach it even if given more days to use the X10 [Figure 3].

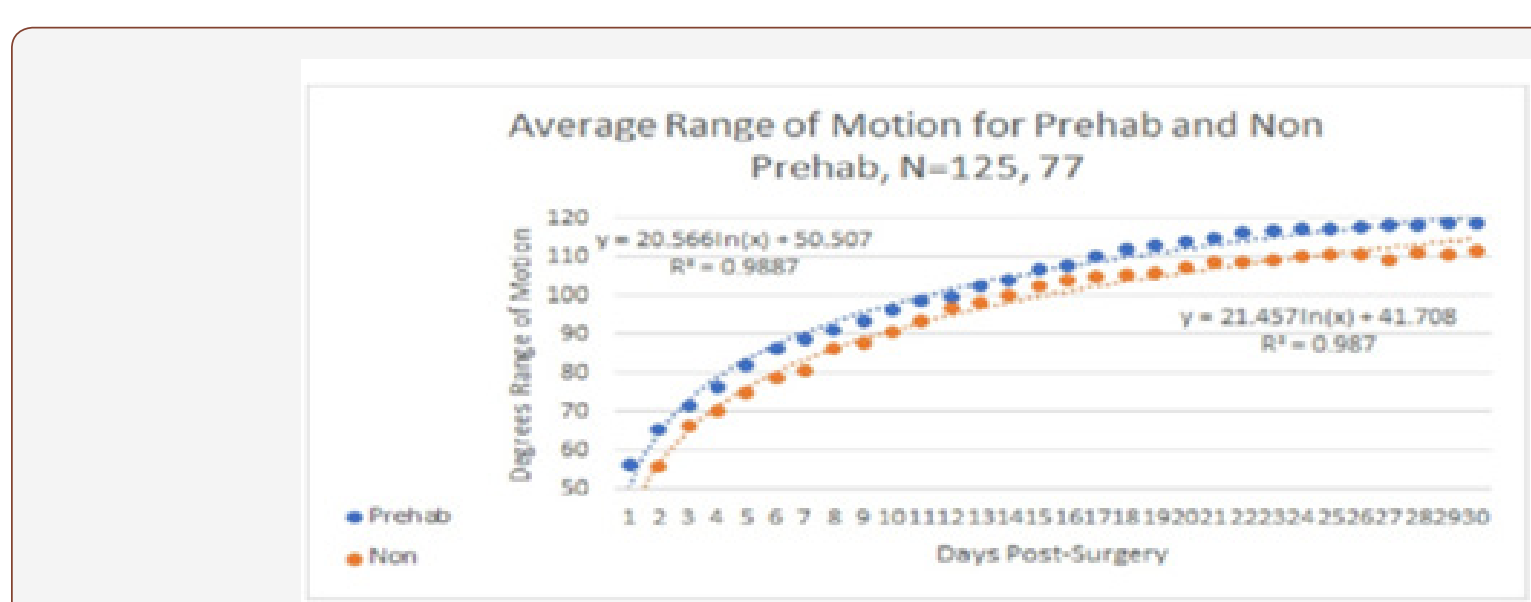

Figure 2: Rehabilitation, mean range of motion of the prehabilitation (treatment) vs non-prehabilitation (control) over time.

\section{Cumulative $\%$ of Patients with RoM $>=110^{\circ}, \mathrm{N}=125,76$}

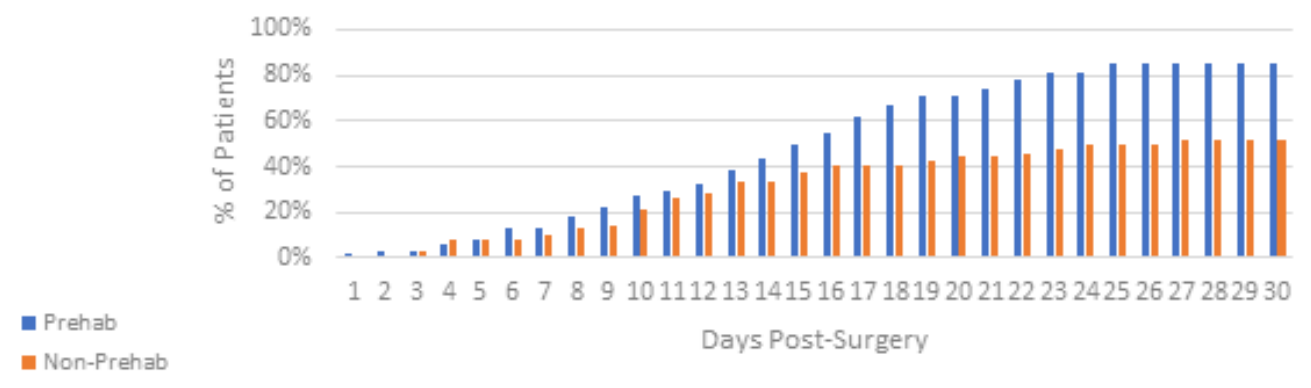

Figure 3: Here we have plotted the cumulative frequency of patients who reached $110 \AA$ RoM for patients from the two treatment groups. While the mean RoM differed by less than 9 degrees between the two groups, the cumulative frequency differed by more than $30 \%$.

\section{Strength}

On average, patients who had prehabilitation gained quadriceps strength compared to baseline (table 2), whereas those who did not have prehabilitation lost quadriceps strength relative to their baseline. We conducted a repeated measure ANOVA and found a significant time $\mathrm{x}$ treatment interaction $\left.\left(\mathrm{F}_{1,83}=3.99, \mathrm{P}<0.05\right)\right)$ indicating that the two groups did not respond the same way. Either treatment is superior to the one month $50 \%-67 \%$ strength deficit reported in the literature $[4,14]$.

If prehabilitation improved hamstring strength, we would expect to find a significant treatment-by-time interaction. While,we did observe that the prehabilitation group had an increase in hamstring strength relative to their baseline score $(10 \%)$ and that the non-prehabilitation group did not even recover their presurgical strength but the interaction was not significant $\left(\mathrm{F}_{1,83}=2.008\right.$, $\mathrm{P} 0<0.16$ ) (Table 2).

Patients in the prehabilitation group had an average increase in post-surgical calf strength, relative to baseline (30\%). The time-bytreatment interaction was significant $(\mathrm{F} 1,81=8.52, \mathrm{P}<0.005)$ (table 2). 
Table 2: In this table, we compare the two treatments at baseline vs final measurement. There was no significant difference between the response to the treatment over time for hamstrings but there was a significant time by treatment interaction for quadriceps and calf strength.

\begin{tabular}{|c|c|c|c|c|c|c|c|c|}
\hline & \multirow[b]{2}{*}{ Treatment } & \multicolumn{2}{|c|}{ Baseline } & \multicolumn{2}{|c|}{1 Month Post Surgery } & \multirow[b]{2}{*}{$\mathrm{N}$} & \multirow[t]{2}{*}{$\begin{array}{c}\text { Interaction } F \\
1,81 \\
\end{array}$} & \multirow[t]{2}{*}{$\boldsymbol{P}$} \\
\hline & & Mean (kg) & St. Dev & Mean (kg) & St. Dev & & & \\
\hline \multirow{2}{*}{ Quadriceps } & Prehabilitation & 23.1 & 7.7 & 24.4 & 6.8 & 77 & \multirow{2}{*}{4.00} & \multirow{2}{*}{$<0.05$} \\
\hline & Non Prehabilitation & 27.9 & 4.5 & 23.2 & 10.0 & 8 & & \\
\hline \multirow{2}{*}{ Hamstrings } & Prehabilitation & 17.1 & 6.0 & 18.8 & 3.5 & 77 & \multirow{2}{*}{2.01} & \multirow{2}{*}{ N.S. } \\
\hline & Non Prehabilitation & 14.7 & 6.3 & 13.7 & 4.9 & 8 & & \\
\hline \multirow{2}{*}{ Calf } & Prehabilitation & 48.3 & 16.1 & 63.0 & 13.4 & 77 & \multirow{2}{*}{8.24} & \multirow{2}{*}{0.005} \\
\hline & Non Prehabilitation & 56.2 & 17.9 & 56.3 & 22.6 & 8 & & \\
\hline \multirow[t]{2}{*}{ TUG } & Prehabilitation & 9.6 & 3.1 & 9.5 & 2.8 & 86 & \multirow{2}{*}{12.55} & \multirow{2}{*}{0.001} \\
\hline & Non Prehabilitation & 9.5 & 2.8 & 12.2 & 3.3 & 19 & & \\
\hline
\end{tabular}

Timed Up and GO Test (TUG test)

On average patients who used the X10 for prehabilitation improved their TUG test score by 0.1 seconds (1\%), indicating

\section{Comparison to Calatayud et al. 4, table 3}

Table 3: A comparison of multiple times between X10 prehabilitation and rehabilitation and Calatayud et al. 2017. Both studies measured range of motion parameters and quadriceps and hamstring strength before and one month after surgery. The most important findings were that at 30 days post-surgery the $\mathrm{X} 10$ prehabilitation group had superior extension, flexion, quadriceps and hamstring strength compared to the Calatayud control and interventiongroup.

Table 3: Comparison to Calatayud et al. 2017

\begin{tabular}{|c|c|c|c|c|c|c|c|c|}
\hline & & & \multicolumn{2}{|r|}{$\mathrm{X} 10$} & \multicolumn{4}{|c|}{ Calatayud et al. } \\
\hline & & & & & Control & & Intervention & \\
\hline & & & Mean & CI & Mean & CI & Mean & CI \\
\hline \multirow[t]{3}{*}{ Extension } & \multirow{3}{*}{$\circ$} & Baseline & 10.2 & 8.9-11.6 & 14 & $13.0-15.0$ & 14.4 & $13.9-16.0$ \\
\hline & & Pre-surgery & 1.4 & $0.7-2.2$ & 14.9 & $13.9-16.0$ & 6.6 & $5.6-7.6$ \\
\hline & & 1 Month Post & 0.2 & $0.0-0.3$ & 16.9 & $15.9-17.9$ & 11.1 & $10.1-12.2$ \\
\hline \multirow[t]{3}{*}{ Flexion } & \multirow{3}{*}{$\circ$} & Baseline & 110.6 & $108.0-114.0^{\prime}$ & 104.2 & $100.5-107.4$ & 104 & $100.5-107.4$ \\
\hline & & Pre-surgery & 126 & $124.8-127.2$ & 102.8 & $99.3-106.3$ & 114.4 & $110.9-117.8$ \\
\hline & & 1 Month Post & 119.4 & $117.4-121.4$ & 82.3 & $78.8-85.8$ & 88.8 & $85.4-92.3$ \\
\hline \multirow[t]{3}{*}{ Quadriceps } & \multirow{3}{*}{ kg } & Baseline & 23.2 & $21.9-24.5^{\prime}$ & 23.5 & $20.3-26.7$ & 23.5 & $20.4-26.6$ \\
\hline & & Pre-surgery & 28.1 & $27.1-29.1$ & 22 & $18.8-25.2$ & 37.8 & $34.7-0.9$ \\
\hline & & 1 Month Post & 23.2 & $22.8-25.9$ & 7.7 & $4.5-10.9$ & 8.9 & $5.8-12.0$ \\
\hline \multirow[t]{3}{*}{ Hamstring } & \multirow{3}{*}{ kg } & Baseline & 14.8 & 13.8--15.8 & 9.1 & $8.5-9.7$ & 9.2 & $8.7-9.8$ \\
\hline & & Pre-surgery & 19.1 & 18.1-20.2' & 8.2 & $7.6-8.8$ & 17.6 & $17.1-18.2$ \\
\hline & & 1 Month Post & 18.8 & $18.0-19.6$ & 3.9 & $3.3-4.4$ & 8.7 & $8.1-9.3$ \\
\hline
\end{tabular}

The extension of the Calatayud et al. patients, at base line, was worse than that of the X10 patients: The means differ and the two confidence intervals do not overlap. On average the Calatayud et al. patients in the control group had about the same average extension at the pre-surgery time point as they did at their baseline point; while their intervention group had improved $7.8^{\circ}$. The X10 patients also improved $7.8^{\circ}$ from baseline to pre-surgery. The major difference occurred post-surgically, the X10 patients improved to an average of 1.2 degrees of extension 30 days post-surgically, while the Calatayud et al. patients in the control worsened to $16.9^{\circ}$ that they return to their baseline, whereas those who did not use the X10 for prehabilitation were 2.7 seconds slower (28\%). The interaction between the time and treatment was significant $\left(\mathrm{F}_{1,101}=5.89, \mathrm{P}<0.017\right)$. extension and their intervention group worsened to $11.1^{\circ}$ extension 30 days post-surgically.

The pattern for flexion was similar. The critical result was that at 30 days post-surgery, the X10 patients had an average flexion of $119.4^{\circ}$, which was better than the baseline. However, the Calatayudet al., control group had an average flexion of 82.3 degrees which was worse than the baseline, and the intervention group had an average flexion of $88.8^{\circ}$. While the intervention group was better than the control, it was worse than their baseline [15-18]. 
The major story, of the comparison deals with quadriceps strength. Both the Calatayudet al. control and intervention group, lost appreciable post-surgical quadriceps strength, relative to the baseline (67\% and $62 \%$ respectively). In contrast, the prehabilitation X10 treatment resulted in patients fully regaining their baseline strength 30 days post-surgery. Similarly, the X10 patients on average had better hamstring strength 30 days postsurgery (27\% improvement) than at their baseline, while the Calatayud et al. patients had worse hamstring strength at 30 days post-surgery. This was particularly true for patients in the control group (57\% decline). However, the Calatayud et al. intervention group had a 1.6 second improvement in their TUG test, while the X10 group only improved by 0.1 second on average over their baseline.

\section{Discussion}

The literature on the efficacy of prehabilitation is mixed [18], and has predominately relied upon relatively small studies. Here, we examined the results for 201 patients and found that X10 prehabilitation improved RoM at 30 days post-surgery by an average of $8.5^{\circ}$ greater than non-prehabilitation patients. Relative to their baseline value before surgery, prehabilitation patients gained an average of $12^{\circ}$ RoM. These contrasts sharply with the results of Calatayud et al. [4] who reported a decline of $22^{\circ}$ relative to baseline at one-month post-surgery. The average Calatayud et al patient, at one-month post-surgery had $82.3^{\circ}$ RoM, whereas the $\mathrm{X} 10$ prehabilitation patients had an average RoM of $120.4^{\circ}$.

McClelland et al. [15] report that patients use less than their full RoM when performing tasks such as squatting and lunging that require weight bearing. This is likely the result of strength deficits. Such strength deficits have been reported using before and after data [4], comparing the effected leg to the unaffected leg [14], and comparing affected leg strength to that of healthy controls [16]. Using a before and after comparison, 4 Calatayud et al. found a substantial deficit at one-month post-surgery (62\% deficit) while Huang et al. [17], using a comparison to healthy adults, found a $30 \%$ deficit an average of 10.4 years after surgery. Clearly, leg strength, and particularly quadriceps strength is a critical issue in a total knee arthroplasty recovery. Catalayud et al. [4] also reported a decline in both quadriceps (62\%-67\%)and hamstring strength( $20 \%-46 \%$ )post-surgery relative to their baselines. In contrast, X10 prehabilitation resulted in $20 \%$ increase in quadriceps strength relative to baseline and a 10\% gain in hamstring strength. Walls et al. [5] found that NIMS prehabilitation improved quadriceps strength, but the difference between patients who had prehabilitation and those that didn't only became apparent at 6-12 weeks post-surgery while, with the X10, the difference was quite apparent at two to three weeks post-surgery.

Using the TUG test as a functional measure of strength, Cavill et al. [9] report that prehabilitation improved post-surgical flexion but that this did not translate into a functional difference between their prehabilitation and control group. Here we found that the prehabilitation treatment resulted in patients returning to their presurgical baseline TUG test values, while non-prehabilitation patients were $28 \%$ slower than their baseline values. Thus, X10 prehabilitation provides a functional gain in knee strength.

In addition to assessing strength, Brown et al. [1] assessed patients' perceptions of their physical, emotional, social and mental health compared to population norms. They found that prehabilitation enhanced emotional and social well being as well as the perception of physical functioning. Compared to their control, participants in prehabilitation had a 69\% higher score for physical functioning, a $17 \%$ higher score for general health, a $27 \%$ higher rating for social wellbeing, and a $20 \%$ higher score for emotional wellbeing, indicating that prehabilitation improves more than strength and RoM.

Several factors contribute to the success of X10 prehabilitation. As mentioned before, the mechanism of the machine safeguards patients from pain so patients are not fighting the therapy. Second because the machine provides instant digital feedback to the patient, they always know where they are, and where they have been. Patients find this highly motivating and compete with themselves to improve. With telemedicine coaching, patients can reach their coach at any time, and most coaches check in daily with their patients, providing another source of motivation. Our results, coupled with Brown et al. [1], suggest that patients who have prehabilitation may feel more in control of their recovery and may have a greater sense of what they can and cannot do with a compromised knee.

Limitations: There are two major limitations to our study: 1) We did not directly compare the X10 to standard physical therapy, and 2) we did not engage in long term follow up. Nevertheless, the results do show that $\mathrm{X} 10$ prehabilitation is superior to no X10 prehabilitation and comparisons with the literature strongly indicate that X10 prehabilitation is superior to reports in the literature.

\section{Conclusion}

We have demonstrated that X10 prehabilitation improves the extension, flexion, range of motion, quadriceps strength, hamstring strength and calf strength prior to surgery. Patients who participated in X10 prehabilitation outperformed, post-surgically, patients who had the X10 for rehabilitation, but who did not participate in X10 prehabilitation.

\section{Acknowledgement}

We thank Halley Orthopedics for providing us access to their data, and Mike McClellan for assistance with the data.

\section{Appendix}

The X10 measures the angle of the activator arm using an ultrasensitive inclinometer. To establish the relationship between 
the angle of the actuator arm and the angle of a patient's leg, we had three threrapist measure the 13 different angles on three patients using a digital goniometer. The data were analyzed utilizing a stepwise multiple linear regression, with the subject, the assessor, and angle as variables. Only the angle entered into the equation, accounting for $99.6 \%$ of the variance. Importantly, the slope was 0.982 and the intercept was -1.668 indicating that the machine slightly under-estimated the angle, amounting to an underestimate of 4 degrees at $130^{\circ}$. Thus, we will slightly underestimate the true amount of flexion especially at higher angles. Similarly, we will slightly underestimate extension by 2-3 degrees. These discrepancies are likely due to the difficulties of measuring the angle of the leg while seated on the machine, rather than due to a discrepancy in the machine's measurement.

We also compared the output from the machine's load cell, used to measure the force applied to the actuator's arm by the patient's leg, to known weights. We used 10 different weights ranging from 0 to $130 \mathrm{lbs}$. Each weight was measured three times, with no variation among trials. The coefficient of determination (R2) was $99.99 \%$ and the slope was 0.9879 with an intercept of 0.072 indicating that the machine accurately reflects strength measurements.

\section{Conflicts of Interest}

No conflicts of interest.

\section{References}

1. Brown K., Topp R, Brosky JA, Lajoie AS (2012) Prehabilitation and quality of life three months after total knee arthroplasty: a pilot study. Perceptual \& Motor Skills: Physical Development \& Measurement 115: 765-774.

2. Fortin PR, Clarke AE, Joseph L, Liang MH, Tanze M, et al. (1999) Outcomes of total hip and knee replacement:Preoperative functional status predicts outcomes at six months after surgery. Arthritis \& Rheumatism 42(8): 1722-1728.

3. Van Leeuwen, DM, De Ruiter CJ, Nolte, PA, De Haan A (2014) Preoperative strength training for elderly patients awaiting total knee arthroplasty. Rehabilitation Research and Practice.

4. CalatayudJ, Casaña J, Ezzatvar Y, Jakobsen, Sundstrup E et al. (2017) High-intensity preoperative training improves physical and functional recovery in the early post-operative periods after total knee arthroplasty: a randomized controlled trial. Knee Surg Sports Traumatol Arthrosc

5. Beaupre LA, Lier D, Davies DM, Bill Johnston (2004) The effect of a preoperative exercise and education program on functional recovery, health related quality of life, and health service utilization following primary total knee arthroplasty. The Journal of Rheumatology 31: 11661173.

6. Rooks, DS, Huang IJ, Bierbaum BE, Bolus SA Rubano, J Connoly, CE, et al. (2006) Effect of Preoperative Exercise on Measures of Functional Status in Men and Women Undergoing Total Hip and Knee Arthroplasty. Arthritis \& Rheumatism 55: 700-708.

7. Swank AM, Kachelman, JB, Bibeau, W, Quesada, PM, Nyland, H, et al (2011) Prehabilitation before total knee arthroplasty increases strength and function in older adults with severe osteoarthritis. Journal of Strength and Conditioning Research 25(3): 318-325.

8. Cavill, S, McKenzie, K, Munro A, McKeever J, Whelan, L et al. (2016) The effect of prehabilitation on the range of motion and functional outcomes in patients following the total knee or hip arthroplasty: A pilot randomized trial. Physiotherapy Theory \& Practice 32(4): 262-270.

9. Walls, RJ, McHugh G, Gorman DJ, Moyna NM, O Bryne (2010) Effects of preoperative neuromuscular electrical stimulation on quadriceps strengthand functional recovery in total knee arthroplasty. A pilot study. BMC Musculoskeletal Disorders 11: 119-128.

10. Jahic D, Omerovic D, Tanovic AT, Dzankovic F, Campara MT (2018) The effect of prehabilitation on postoperative outcome in patients following primary total knee arthroplasty. Medical Archive 72(6): 439-443.

11. Chen H, Li S, Ruan T, Liu L, Fang L (2018) Is it necessary to perform prehabilitationexercise for patients undergoing total knee arthroplasty: meta-analysis of randomized controlled trials.The Physician and Sports Medicine 46(1): 36-43.

12. Roubal PJ, Freeman DC (2018) Are computer controlled, pressure modulated knee rehabilitation machines valuable following knee arthroplasty? Global Journal of Orthopedics Research 1: 1-8

13. Rowe PJ, Myles CM, Nutton R (2005) The effect of total knee arthroplasty on joint movement during functional activities and joint range of motion with particular regard to higher flexion users. Journal of Orthopaedic Surgery 2005 Hong Kong 13: 13-18.

14. Townsend LA, Roubion RC, Devin M, Bourgeois DM, Leonardi C, et al.(2018) Impact of age on patient reported outcome measures in total knee arthroplasty. J Knee Surg 31: 580-584.

15. Mizner RL, Petterson SC, Snyder Mackler L (2005) Quadriceps strength and the time course of functional recovery after total knee arthroplasty. Journal of Orthopaedic\& Sports Physical Therapy 35(7): 424-436.

16. McClelland JA, Feller JA, Menz HB, Webster, KE (2017) Patients with total knee arthroplasty do not use all of their available range of knee flexion during functional activities. Clinical Biomechanics 43: 74-78.

17. Bade, MJ, Kohrt, WM, Stevens-Lapsley JE, 2010 Outcomes before and after total kneearthroplasty compared to healthy adults. Journal of Orthopaedic \& Sports Physical Therapy 40(9): 559-567.

18. Huang CH, Cheng CK, Lee YT, Lee KS (1996) Muscle strength after successful total knee replacement. A 6- to 13-Year follow up clinical orthopaedics and related research 328: 147-154 